\title{
Avaliando as customizações do Moodle para a plataforma MOOC Saviesa
}

Beatriz Brito do Rêgo - SPIDeLab - IME/UFBA - beatrizbr@ufba.br Filipe Adeodato Garrido - SPIDeLab - IME/UFBA - filipe.garrido@ufba.br

Jean C. S. Rosa - SPIDeLab - IME/UFBA- jean.rosa@ufba.br

Ecivaldo de Souza Matos - SPIDeLab - IME/UFBA - ecivaldo@ufba.br

Resumo: MOOC são cursos online, abertos e massivos que buscam democratizar a educação. Entretanto, devido a essas características, eles podem causar altos custos para o seu desenvolvimento e a manutenção. $\mathrm{Na}$ tentativa de reduzir esses custos, alguns pesquisadores sugerem o uso do Moodle como plataforma base, depois de aplicadas algumas adequações. Este artigo apresenta os resultados de um estudo para verificar se o Saviesa, um MOOC desenvolvido sobre o Moodle, atende as orientações básicas de customização de uma plataforma de MOOC. A partir da avaliação foram identificados alguns problemas de interação humano-computador, potencialmente mitigáveis em tempo de design, caso as orientações de customização do Moodle para MOOC sejam consideradas.

Palavras-chave: MOOC; Interação Humano-Computador; Método de Inspeção Semiótica, Moodle.

\section{Evaluating Moodle customization for the MOOC platform Saviesa}

\begin{abstract}
MOOCs are online, open, and massive courses that seek to democratize education. However, due to these characteristics, they can cause high costs for their development and maintenance. Some researchers suggest using Moodle as a base platform to reduce these costs after some adjustments. This article presents results of a study to verify if Saviesa, a MOOC developed from Moodle, satisfies the basic MOOC customization guidelines. We identify some problems of human-computer interaction from the Saviesa evaluation. These problems potentially can be mitigable in interaction design time if the Moodle customization guidelines for MOOC are considered.
\end{abstract}

Keywords: MOOC; Human-Computer Interaction; Semiotic Inspection Method, Moodle.

\section{Introdução}

Diversas abordagens de apoio ao ensino têm sido criadas/utilizadas para melhorar tanto a qualidade quanto a disponibilidade da educação. Uma dessas abordagens é conhecida por Massive Open Online Course (MOOC), cursos online, abertos e em massa, que buscam democratizar a educação, atraindo uma população diversificada de estudantes de ampla faixa etária, formação e nacionalidades (He et al., 2015).

Fassbinder, Delamaro e Barbosa (2014) fazem distinção entre cursos MOOC e plataformas MOOC. A plataforma MOOC corresponde a qualquer ambiente que permita a criação e a hospedagem de um curso MOOC. Por sua vez, o curso MOOC é gratuito, baseado na web, com o registro aberto e currículo compartilhado publicamente (Gené, et al., 2014).

Porém, a viabilidade econômica dos cursos MOOC ainda é algo discutido na literatura, por causa do alto custo para desenvolver e manter uma plataforma MOOC (Bastos, Biagiotti, 2014). Nesse sentido, Do Rêgo, Garrido e Matos (2018) utilizaram o 
Moodle ${ }^{1}$ para a construção de uma plataforma MOOC, a qual chamaram de Saviesa. O Moodle é considerado um dos ambientes virtuais de aprendizagem mais utilizados para suporte a cursos a distância e presenciais nas universidades federais brasileiras (Sabbatini, 2007).

Antes do desenvolvimento do Saviesa, Do Rêgo, Garrido e Matos (2018) construíram e utilizaram um conjunto de orientações para customizar o Moodle, possibilitando sua adequação às plataformas MOOC sob a perspectiva da comunicabilidade, um dos critérios de qualidade de sistemas interativos. Pois, sem customização, o Moodle não atende aos critérios básicos de uma plataforma MOOC (Do Rêgo, Garrido, Matos, 2018). Essas orientações para o design de uma plataforma MOOC foram concebidas a partir da avaliação da comunicabilidade de duas plataformas MOOC: a Coursera $^{2}$ e a $\mathrm{EDx}^{3}$ (Do Rêgo, et al., 2019).

Assim, este artigo apresenta os resultados de um estudo para verificar se o Saviesa atende as orientações básicas de customização de um MOOC, conforme sugerido por Do Rêgo, Garrido e Matos (2018). Nesse sentido, as contribuições deste artigo estão relacionadas à exemplificação e à demonstração da efetividade das orientações para a customização de plataformas Moodle para torná-las plataformas MOOC.

Este artigo está dividido em cinco seções. A próxima seção trata sobre as definições de MOOC e as regras básicas de customização do ambiente Moodle. Em seguida, na terceira seção há descrição da metodologia. A avaliação do Saviesa, com o intuito de verificar sua adequabilidade às regras básicas de customização é apresentada na quarta seção. Por fim, seguem as considerações finais e trabalhos futuros na quinta seção.

\section{Fundamentação teórica}

O termo Massive Open Online Course foi criado no ano de 2008 por Dave Cormier para descrever um determinado modelo de curso online desenvolvido por Stephen Downes e George Siemens (Grainger, 2013). Baloi (2009) acreditava que os MOOC possuíam o objetivo de compartilhar diferentes experiências individuais, em que o estudante, com aquilo que aprendeu em casa, ao se encontrar com colegas, se enriqueceriam com a sua experiência, ocorrendo assim relações de ajuda recíproca, em que um sujeito aprenderia com o outro, sabendo que a individualidade de cada um deve ser respeitada.

Com o tempo, algumas iniciativas de MOOC foram elaboradas com o intuito de apoiar essa nova forma de aprendizado, sendo uma delas a criação de plataformas que proporcionassem suporte aos MOOC (Grainger, 2013). Algumas dessas plataformas são conhecidas mundialmente, como Coursera, EDx e Udacity ${ }^{4}$. No Brasil, alguns exemplos de plataformas de apoio a cursos MOOC são a Lúmina ${ }^{5}$, da Universidade Federal do Rio Grande do Sul, e o Saviesa, da Universidade Federal da Bahia (UFBA).

O design de interação do Saviesa foi desenvolvido a partir da perspectiva de comunicabilidade $^{6}$ da Engenharia Semiótica (De Souza 2005). Para isso, foi utilizada a Modeling Language for Interaction as Conversation ${ }^{7}$ (MoLIC) como ferramenta de

\footnotetext{
${ }^{1}$ https://moodle.org/?lang=pt_br

2 https://pt.coursera.org/

${ }^{3}$ https://www.edx.org/

${ }^{4}$ https://br.udacity.com

${ }^{5}$ https://lumina.ufrgs.br/

${ }^{6}$ Entendendo-se que quando um usuário consegue compreender como o sistema funciona, é porque o designer se expressou adequadamente através da interface. Ou seja, comunicabilidade é a habilidade de um sistema computacional interativo em comunicar-se (De Souza, 2005).
}

${ }^{7}$ Linguagem para a Modelagem de Interação como Conversa - tradução nossa. 
modelagem da interação. O Moodle foi escolhido como plataforma base para o Saviesa por ser um software de código aberto (open source) e ter baixo custo para sua adequação (Garrido, Do Rêgo, Matos, 2018b).

O Moodle é um ambiente virtual de aprendizagem desenvolvido como software livre e gratuito com o intuito de ser compatível, flexível e de fácil modificação/atualização, permitindo a configuração e customização da interface e dos serviços pelas instituições em instalações próprias (Dougiamas, Taylor, 2003). Nesse sentido, o Moodle tem sido um dos recursos adotados para apoiar a mediação entre os professores e alunos em cursos presenciais e a distância (Delgado, 2009; Torres, 2008, Silva, 2008; Mota et al., 2009).

O Moodle tem sido utilizado por diversas instituições no mundo, havendo uma grande comunidade de usuários e desenvolvedores, cujos membros estão envolvidos em atividades que abrangem desde correções de erros e o desenvolvimento de novas ferramentas à discussão sobre estratégias pedagógicas de utilização do ambiente e suas interfaces (Torres, Silva, 2008).

Do Rêgo, Garrido e Matos (2018) consideram que o Moodle, a partir de customizações, pode servir como uma plataforma MOOC e descreveram orientações para essas customizações, a saber: (i) deve ter uma central de ajuda ou FAQ e facilidade de acesso do sistema, podendo acessar todas as páginas do MOOC, elemento fundamental em plataformas de uso em massa; (ii) deve permitir acesso à plataforma por usuários externos à instituição; (iii) não se deve exigir cadastro às pessoas que desejam usar (ou conhecer) os cursos; (iv) o design instrucional dos cursos deve ser direcionado ao ensino a distância, em vez de apoio ao ensino presencial; (v) deve possibilitar que todos possam ter acesso aos materiais submetidos pelos demais estudantes, de modo a favorecer a construção colaborativa de conhecimento; (vi) haja padronização de botões e sinais de alerta; (vii) deve haver um tópico do fórum no final de cada módulo, para incentivar a interação do aluno; (viii) haja padronização do idioma da plataforma (tradução completa, quando aplicável); (ix) tenha uso de sinais facilmente reconhecíveis comumente usados em outros sistemas (herdados); (x) haja barra de progresso visível na página principal do curso; e (xi) haja planejamento do design instrucional do conteúdo do curso para um MOOC.

$\mathrm{Na}$ próxima seção será apresentada a metodologia aplicada na pesquisa para investigar se o Saviesa pode atender aos requisitos mínimos de uma plataforma MOOC, considerando essas orientações para customização

\section{Metodologia}

O estudo foi dividido em duas etapas: (i) a avaliação da interação do Saviesa por meio do Método de Inspeção Semiótica (MIS) (De Souza, 2005); e (ii) a análise dos resultados sob a perspectiva das orientações de customização do Moodle para plataforma MOOC descritas por Do Rêgo, Garrido e Matos (2018), a fim de propor o redesign de interação da plataforma MOOC, caso fosse necessário.

Considerando a comunicabilidade como atributo de qualidade de interação no desenvolvimento do Saviesa, o MIS foi utilizado para avaliar a qualidade da metacomunicação ${ }^{8}$. A avaliação foi realizada por três estudantes de pós-graduação stricto sensu em Ciência da Computação, com a supervisão de um pesquisador e professor especialista em Interação Humano-Computador e em Engenharia Semiótica.

A avaliação, conforme proposta pelo MIS, ocorreu de forma autoguiada, a partir da análise de três conjuntos de signos, nomeadamente: metalinguísticos, estáticos e dinâmicos.

\footnotetext{
${ }^{8}$ Processo de comunicação entre o designer e usuário por meio da interface do sistema projetado pelo designer.
} 
Os signos metalinguísticos são usados pelos projetistas para comunicar explicitamente aos sujeitos-usuário os significados atribuídos aos demais signos codificados na interface e como eles devem ser usados. Os signos estáticos expressam a condição do ambiente, cujo significado deve ser interpretado não obstante as relações causais e temporais da interface. Por sua vez, os signos dinâmicos expressam o comportamento do sistema, envolvendo aspectos temporais e causais da interface (De Souza, 2005).

A inspeção ocorreu na seguinte ordem: análise dos signos metalinguísticos, estáticos e dinâmicos. Após a análise dos conjuntos de signos, houve a elaboração da mensagem de metacomunicação correspondente de cada uma das três classes de signos. Para inspeção completa da plataforma Saviesa, utilizou-se o curso Práticas pedagógicas para o ensino de informática, em que o conteúdo foi desenvolvido a partir do framework conceitual de design instrucional distribuído para concepção participativa de um curso MOOC (Garrido, Do Rêgo, Matos, 2018a).

Por fim, os avaliadores efetuaram a consolidação dos resultados, em que foram sumarizadas as rupturas de comunicação encontradas no Saviesa. De posse desse resultado do MIS, os pesquisadores estabeleceram relações entre os problemas de interação/comunicabilidade relatados e as orientações propostas por Do Rêgo, Garrido e Matos (2018), a fim de conceber sugestões de melhorias na interação do Saviesa. As sugestões de melhorias foram indicadas a partir das indicações apresentadas no estudo de Do Rêgo, Garrido e Matos (2018) e Do Rêgo, et al., (2019) que apresentam sugestões de melhorias e uma lista de indicações de funções de que um MOOC deve ter, proporcionando assim o seu redesign de interação e validando a utilização do Moodle como plataforma MOOC. Os resultados da aplicação da metodologia são apresentados na próxima seção.

\section{Resultados}

Os resultados da avaliação por meio do MIS são expressos a partir das mensagens de metacomunicação e suas análises. Neste artigo, as mensagens serão apresentadas de forma fragmentada. A primeira versão do Saviesa não foi desenvolvida a partir das orientações definidas por Do Rêgo, Garrido e Matos (2018), mas as orientações foram utilizadas para sua avaliação, juntamente com os resultados do MIS. A seguir, após a apresentação da avaliação, serão apresentadas as sugestões para o redesign da interação da plataforma.

Conforme análise dos signos metalinguísticos, o Saviesa foi idealizado com o intuito de ofertar diversos cursos gratuitos, com o objetivo de ampliar o acesso da sociedade ao conhecimento construído na UFBA (cf. Fragmento 1 da mensagem de comunicação). No menu da plataforma Saviesa, a central de ajuda está nomeada como Ajuda/FAQ, localizada no último menu da página principal. FAQ é termo bastante utilizado em outros sistemas, o que contribui para a facilidade de reconhecimento e acesso antes de o usuário logar ou se cadastrar no sistema. Na página Ajuda/FAQ da plataforma, estão listadas 11 perguntas de ajuda ao usuário no uso do sistema. Além disso, os termos Saviesa e MOOC são apresentados novamente.

Fragmento 1: Você é um estudante que está a procura de um curso gratuito para aprendizado livre, com o objetivo de compreender a sequência necessária do processo de ensino e de aprendizagem.

O menu de Ajuda/FAQ não está de fácil acesso aos seus usuários. Para ir à página de Ajuda/FAQ, o usuário precisa informar o endereço do Saviesa na barra de navegação, pois não existe botão ou menu que possibilite o redirecionamento do usuário para a central de ajuda ( $c f$. Figura 1). A dificuldade de retorno à central de ajuda após o usuário logar no sistema fere a orientação de que a plataforma deve ter uma central de ajuda ou FAQ, 
elemento fundamental em plataformas de uso em massa e que ela possa ser acessada a qualquer local de navegação da plataforma. Uma forma de mitigar essa grave ruptura de comunicabilidade, seria com o reposicionamento do menu Ajuda/FAQ após a caixa de login dos usuários; também poderia modificar o conteúdo da página Ajuda/FAQ, por ser pouco explicativo.



Figura 1. Interface após autenticação do usuário

Na Figura 2, há uma elemento no lado direito superior do cabeçalho da imagem que ao ser clicado disponibiliza ao usuário o botão de login, para que o usuário possa logar no sistema. Não existe um botão para cadastro, como normalmente é apresentado em outros sistemas. Nesse sentido, a plataforma comunica que ao clicar em login, caso o usuário não possua cadastro ele poderá realizá-lo, mas essa informação não está clara, ruptura essa identificada e descrita por meio do Fragmento 2 da mensagem de metacomunicação.

Fragmento 2: Você é um usuário que entende que ao clicar no botão de login será redirecionado para a tela de cadastro. Porque para ter acesso ao curso você deverá se cadastrar na plataforma Saviesa.

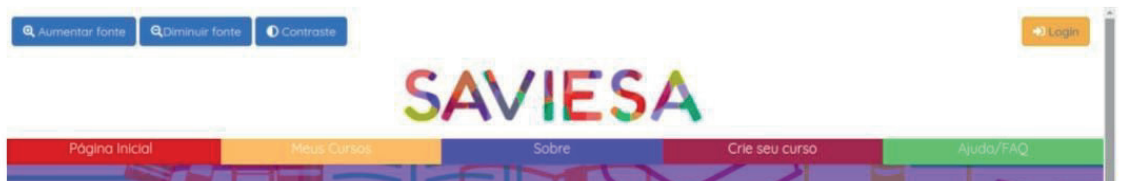

Figura 2. Topo da página principal da plataforma Saviesa

Abaixo dos campos que os avaliadores entenderam ser referente ao login (cf. Figura 3), pois são dois campos de texto sem identificação, há a seguinte frase "Esta é a sua primeira vez aqui?" e um pequeno texto no qual o sistema informa ao usuário a necessidade de criar uma conta para ter acesso ao sistema, logo aos cursos ( $c f$. Figura 3 ). $\mathrm{Na}$ Figura 3 também encontra-se a frase "Para ter acesso completo a este site, você primeiro precisa criar uma conta".

Na plataforma Saviesa está disponível uma opção para que os usuários "visitem" o conteúdo do curso. Mas os signos da interface indicam que o usuário só terá acesso total ao conteúdo do curso se realizar o cadastro. Na página inicial da plataforma há uma mensagem em destaque informando que os cursos disponíveis são gratuitos, o que pode motivar os usuários a realizarem o cadastro.

As rupturas identificadas no trecho apresentado até o momento não atendem às orientações de que a plataforma MOOC deve permitir acesso a usuários externos à instituição e não se deve exigir cadastro às pessoas que desejam usar (ou conhecer) os cursos. Ambas orientações de customização foram associadas, pois na inspeção da plataforma Saviesa foi identificado forte relação entre elas.

Há ruptura de comunicabilidade, dado que botões e sinais de alerta da plataforma não estão padronizados, podendo dificultar o entendimento dos usuários. 
Para ter acesso ao material completo do curso, o usuário terá de se cadastrar na plataforma. Entretanto, conforme Garrido (2018), não é possível customizar o Moodle para atender a essa orientação, dado que o Moodle não permite acesso aos cursos e material disponível sem cadastro prévio, ainda que a entrada seja como "visitante".

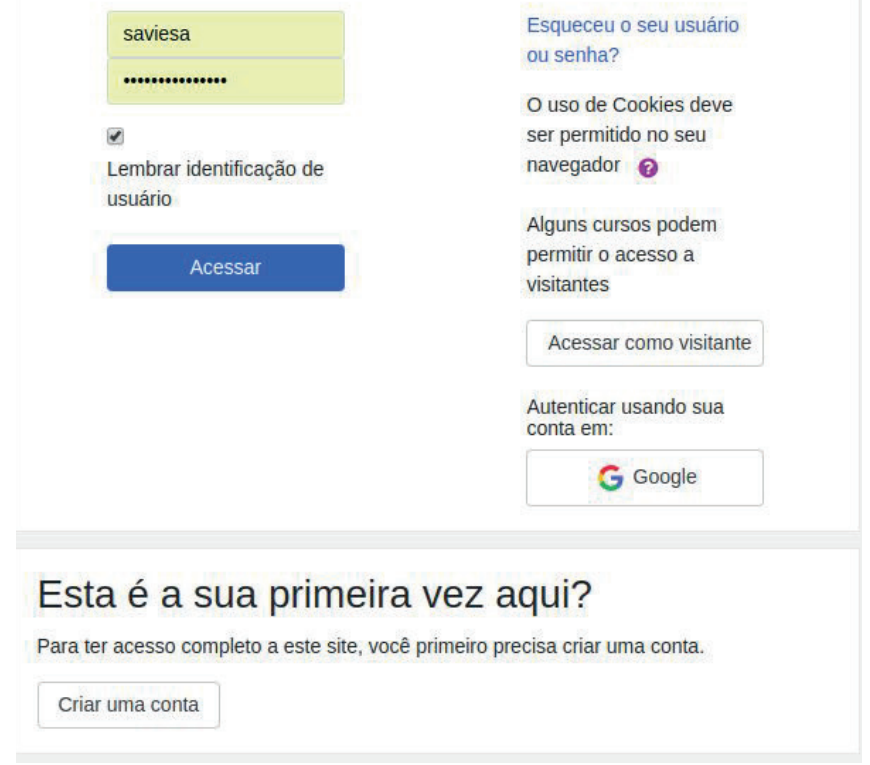

Figura 3. Página de cadastro

No Saviesa é apresentada uma imagem indicando qual é o tipo de conteúdo que será apresentado ao usuário ao clicar na página, como apresentado na Figura 4. Quando a página contém conteúdo textual, apresenta-se uma imagem de duas folhas de papel escritas; quando o conteúdo da página é um vídeo, apresenta-se uma imagem de duas telas com o botão de play. O "símbolo" de play é normalmente utilizado em sistemas de reprodução de vídeo, em conformidade com o conteúdo disponível na página. Por fim, o símbolo de representação dos fóruns são dois balões, elemento visual bastante utilizado para representar diálogos. A partir dessa percepção foi composto o Fragmento 3 da mensagem de metacomunicação.

Fragmento 3: Você é um usuário que utiliza de conhecimento prévio de outras plataformas para entender os símbolos que estão impressos na interface, mas isso não o influencia no entendimento dos símbolos que diferem dos normalmente utilizados.

A plataforma Saviesa possibilita a seus usuários facilidade de interação entre os pares. A criação de um tópico ou resposta utiliza o mesmo formato, em que o sistema apresenta dois campos para que o usuário insira "Seu assunto" e "Digite sua postagem". Abaixo das caixas de texto está o botão para inserção de anexo, diminuindo os passos normalmente pré-definidos em outras plataformas de cursos online. Isto foi descrito no Fragmento 4.

Fragmento 4: Para você foi criado uma plataforma de fácil interação e troca de mensagens entre seus pares. Uma plataforma que disponibiliza mensagens claras para fácil compreensão de seus estudantes. Mas com algumas melhorias necessárias a serem implementadas na plataforma.

No procedimento de anexação de documento foi identificada uma ruptura de comunicação, dado que o título do arquivo aparece ao lado do botão, mas a opção de remoção do arquivo não foi disponibilizada ao usuário. Há ainda outra ruptura nessa mesma tela, ao não haver sinalização de obrigatoriedade dos campos "assunto" e "mensagem". 


\section{Texto}

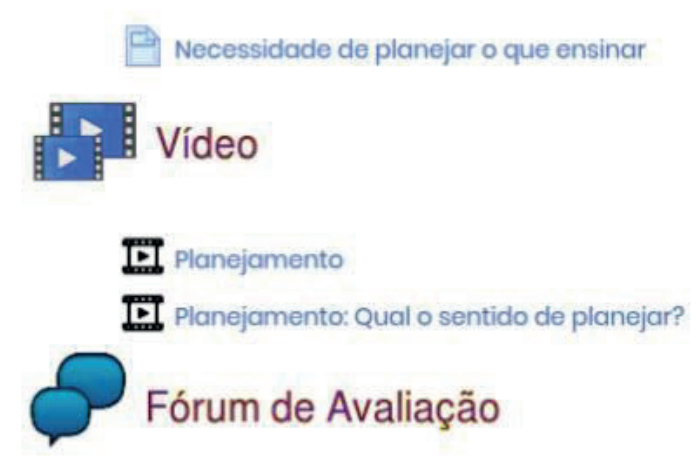

Figura 4. Signos das páginas do curso

Ao clicar no botão referente ao envio da mensagem, o sistema informa que "Ocorreu um erro com o seu envio: A mensagem é necessária". Sendo necessário o destaque para informar que o preenchimento do campo de mensagem é obrigatório ( $c f$. Figura 5). Mas apesar de apenas o preenchimento da mensagem ser obrigatório, ao enviar uma resposta sem preencher o campo referente ao assunto, o sistema automaticamente apresenta a mensagem $\mathbf{R e}^{9}$ : "Pense em uma atividade cotidiana que precisa planejar para alcançar determinados objetivos e descreva a relevância que esse ato lhe proporcionou. Conte sobre a estratégia utilizada".

No momento em que o usuário insere algum conteúdo no campo referente ao assunto para postagem no fórum e confirma o envio, o sistema apresenta o assunto inserido pelo usuário, o conteúdo do campo de mensagem e a seguinte expressão "Sua postagem foi adicionada com sucesso".

Uma possível solução para as rupturas em que a obrigatoriedade de campos ou a possibilidade de remover anexo após inserido não são informadas, seria inserir signos que demonstrem quais campos são obrigatórios, com a possibilidade de remoção de anexo quando utilizada pelo usuário.

Notou-se, por meio da inspeção, que a plataforma oferece suporte apenas ao ensino a distância, como deve ser obrigatório em MOOC. Apesar de algumas rupturas terem sido identificadas, nenhuma delas esteve associada à orientação de que o design instrucional dos cursos deve ser direcionado ao ensino a distância, em vez de apoio ao ensino presencial. Assim, para identificar se o conteúdo está direcionado ao ensino a distância, outro estudo deve ser realizado, específico para avaliação do design instrucional.

Fragmento 5: Você é um usuário que está à procura de aprendizado livre, por meio

de cursos online e a distância que possibilitem o compartilhamento de experiências entre seus pares.

Na Figura 6, o botão de confirmação do campo de pesquisa de tópicos está destacado. Por meio dele, o usuário poderá pesquisar sobre o assunto desejado. Esse botão é nomeado de "Pesquisar", diferentemente de outros botões de confirmação de pesquisa de outras plataformas. Outro signo destacado na Figura 6 é a etiqueta na cor amarela com a palavra "Novo", demonstrando a existência de um novo tópico que não foi visualizado pelo usuário.

Como se trata de um curso MOOC, que poderá ter uma quantidade expressiva de usuários, essa etiqueta pode ajudar os usuários a identificarem quais tópicos não foram visualizados. No primeiro momento foi identificado que a etiqueta era apenas informativa,

\footnotetext{
${ }^{9}$ A sigla "Re" serve para indicar que é uma resposta à questão apresentada no topo da página do fórum.
} 
mas ao passar o mouse em cima da etiqueta percebeu-se que a etiqueta é um botão, cujo clique redireciona o usuário ao "novo" tópico criado. Mas seria melhor que fosse um botão para que os usuários pudessem clicar e ter apenas os novos tópicos que não foram visualizados em seu acesso, no caso o(s) último(s) a não ser visualizado pelo usuário. Esse elemento confuso permitiu a escrita do Fragmento 6.

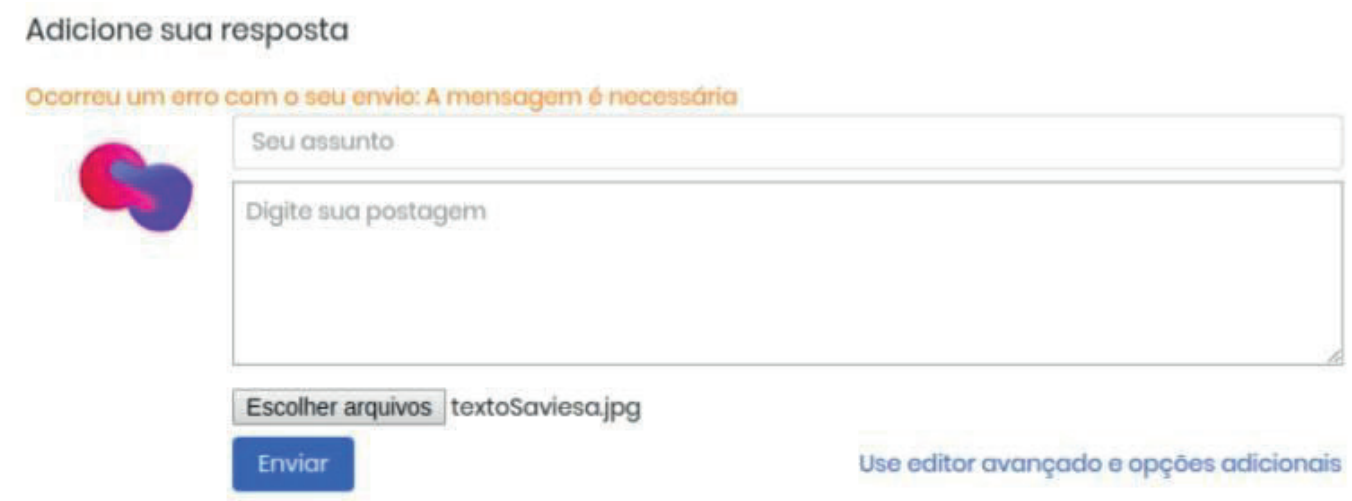

Figura 5. Adição de resposta de fórum

Fragmento 6: Mas quem não entende algumas funções da plataforma irá ignorar por não saber como proceder.

$\mathrm{Na}$ página do fórum encontra-se disponível a opção de ordenação da lista de tópicos, contida em um campo para seleção. Mas ao clicar no campo são apresentadas três opções, com as informações de chave e parâmetro para ordenação da lista de tópicos. Dessa forma, apenas usuários com conhecimento técnico em desenvolvimento de sistemas poderão entender as opções disponibilizadas, como está destacado em vermelho na Figura 6. Essa é uma ruptura grave, em se tratando de um MOOC que poderá ter milhares de discussões criadas, dificultando o acesso do usuário que deseja realizar uma busca a uma discussão específica.

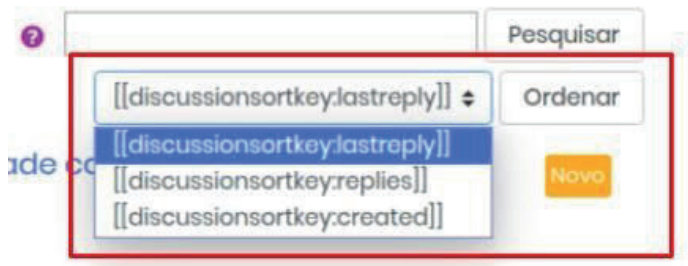

Figura 6. Campo de pesquisar por tópico e ordenação

A orientação de que se deve possibilitar que todos possam ter acesso aos materiais submetidos pelos demais estudantes, de modo a favorecer a construção colaborativa de conhecimento, está relacionada ao conteúdo do curso que evoluirá por meio da colaboração dos usuários por meio dos fóruns. Nas rupturas encontradas nota-se que o usuário que desejar ordenar as discussões não terá sucesso - um erro grave - fazendo com que o Saviesa não alcance a última orientação.

Como sugestão de remodelagem, indica-se a inclusão de uma lista de opções de ordenação, como "por tema", pois por se tratar de um MOOC poderá ter se suportar um grande conjunto de usuários e, por isso, uma quantidade acentuada de diferentes assuntos, o que facilitará a busca dos usuários por um determinado tópico.

O problema mais crítico identificado na plataforma foi a falta de uma central de ajuda com mais informações e acessível após login, para que o estudante possa ter acesso em qualquer lugar que esteja no MOOC, caso ele/ela tenha dificuldade. Mas isso não impossibilita o uso do Moodle como plataforma MOOC, apenas é necessário pequenas 
atualizações para minimizar os problemas de uso pelos estudantes.

\section{Considerações finais e trabalhos futuros}

Neste artigo foi apresentada uma avaliação da interação humano-computador do Saviesa, uma proposta de MOOC por meio de customização do Moodle, com o objetivo de identificar problemas de interação e apresentar uma proposta de redesign considerando os itens de customização apresentadas por Do Rêgo, Garrido e Matos (2018) e validação da customização do Moodle como plataforma MOOC.

A adoção do MIS como mecanismo de avaliação do Saviesa surgiu da intenção de "dar voz" ao usuário por intermédio do avaliador, que assume o papel do primeiro e, por meio das interpretações dos signos presentes na interface, direciona a avaliação sob a perspectiva de abranger as mais variadas personas que possivelmente farão uso do Saviesa. Desse modo, as sugestões de redesign do ambiente MOOC seguem os princípios sugeridos por Do Rêgo, Garrido e Matos (2018) com atenção especial para conceber um ambiente com comunicabilidade adequada e que não cause rupturas no processo de aprendizagem dos usuários.

Com essa avaliação, verificou-se que a plataforma Saviesa atendeu as orientações de customização indicadas por Do Rêgo, Garrido e Matos (2018), havendo apenas dificuldade de acesso à central de ajuda após login ou cadastro no sistema. Por outro lado, identificou-se rupturas de comunicabilidade que poderão ser prejudiciais à interação dos usuários com a plataforma.

A partir desses problemas de interação com sugestões de ajustes/melhorias, apresentados na seção anterior, espera-se constituir diretrizes de (re)design que instruam a concepção de um ambiente de MOOC, favorecendo a colaboração entre pares e o diálogo midiatizado pelo sistema. Nesse sentido, o principal resultado apresentado é a confirmação que o Moodle pode ser utilizado como plataforma MOOC, após seguir orientações de customização definidas por (Do Rêgo, Garrido, Matos, 2018).

Como trabalhos futuros, serão realizadas as adequações no Saviesa a partir das sugestões propostas e um novo ciclo de avaliação para também avaliar a eficácia da aplicação metodológica (i.e., a combinação do MIS e das orientações). Além disso, as orientações propostas por Do Rêgo, Garrido, Matos (2018) serão avaliadas por profissionais de desenvolvimento de software educacional para avaliação de sua qualidade pedagógica. Por fim, espera-se conhecer os impactos dessas modificações pela voz dos usuários.

\section{Agradecimentos}

Agradecemos aos membros do SPIDeLab (Semio-Participatory Interaction Design Lab), ao Grupo de Pesquisa e Extensão em Informática, Educação e Sociedade - Onda Digital e à Coordenação de Aperfeiçoamento de Pessoal de Nível Superior (CAPES) pelo financiamento desta pesquisa por meio do Código 001.

\section{Referências}

BALOI, J. A. A concepção da educação democrática na obra "democracia e educação" de Jonh Dewey. Monografia. Faculdade de Filosofia e Ciências Humanas da Universidade São Tomás de Moçambique, Maputo. 2019.

BASTOS, R. C.; BIAGIOTTI, B. MOOCs: uma alternativa para a democratização do ensino. RENOTE - Revista Novas tecnologias na Educação, Porto Alegre, v.12, n.1, p. 65-80, 2014.

DELGADO, L. M. M. Uso da plataforma Moodle como apoio ao ensino presencial: um 
estudo de caso. Programa Interdisciplinar de Linguística Aplicada. Faculdade de Letras-UFRJ. 2009.

DE SOUZA, C. S. The semiotic engineering of human-computer interaction. Massachusetts (EUA): MIT Press. p. 3122005.

DO RÊGO, B. B., GARRIDO, F., MATOS, E. Moodle como ambiente MOOC: orientações para o redesign de interação. Revista Novas Tecnologias na Educação - RENOTE. v. 16, n. 1. 2018.

DO RÊGO, B. B., GARRIDO, F., ROSA, J., MATOS, E. Communicability problems on MOOCs: a study around metacommunication. In: Simpósio Brasileiro de Informática na Educação (SBIE). Anais. p. 1261-1270. 2019.

DOUGIAMAS, M.; TAYLOR, P. Moodle: Using learning communities to create an open source course management system. In: EdMedia: World Conference on Educational Media and Technology, 2003, Honolulu/Hawaii (USA). Anais. Waynesville: Association for the Advancement of Computing in Education (AACE). p. 171-178. 2003.

FASSBINDER, A., DELAMARO, M. E., BARBOSA, E. F. Construção e uso de MOOCs: uma revisão sistemática. In: Brazilian Symposium on Computers in Education (Simpósio Brasileiro de Informática na Educação-SBIE). Anais. p. 332-341. 2014.

GARRIDO, F., DO RÊGO, B. B., MATOS, E. Modelando a Interação (Humano-Computador) de um Fórum de Discussão para MOOC: MoLIC em uso. Revista Novas Tecnologias na Educação - RENOTE. v. 16, n. 2, p. 321-330. 2018a.

GARRIDO, F., DO RÊGO, B. B., MATOS, E. Design instrucional orientado a artefatos: uma abordagem participativa e distribuída. In: Anais do Simpósio Brasileiro de Informática na Educação (SBIE). Anais. p. 258-267. 2018b.

GRAINGER, B. Introduction to moocs: avalanche, illusion or augmentation. Policy BriefUNESCO (july). Institute for Information Technologies in Education. 2013. Disponível em: $<$ http://iite.unesco.org/pics/publications/en/files/3214722.pdf $>$ Acesso em 03 abr. 2018.

GENÉ, ORIOL BORRÁS; NÚÑEZ, MARGARITA MARTÍNEZ; BLANCO, ÁNGEL FIDALGO. Gamification in MOOC: Challenges, Opportunities and Proposals for Advancing MOOC Model. In: Proceedings of the Second International Conference on Technological Ecosystems for Enhancing Multiculturality. ACM. [s/p]. 2014.

HE, J., BAILEY, J., RUBINSTEIN, B. I., E ZHANG, R. Identifying at-risk students in massive open online courses. In: AAAI, p. 1749-1755. 2015.

MOTA, R.; INMORATO, A. Mooc, uma revolução em curso. Jornal da ciência, São Paulo, nov. 2012. Disponível em: $<$ http://www.jornaldaciencia.org.br/Detalhe.jsp?id=85111 $>$. Acesso em 20 mai. 2018.

SABBATINI, R. M. Ambiente de ensino e aprendizagem via internet: a plataforma Moodle. 2007. Instituto EduMed, v.25, [s/p] 2007. Disponível em:

$<$ http://www.ead.edumed.org.br/file.php/1/PlataformaMoodle.pdf> Acesso em 03 abr. 2018.

TORRES, A. A,. SILVA, M. O ambiente Moodle como apoio à educação a distância. In:

Simpósio Hipertexto e Tecnologias na Educação. Multimodalidade e Ensino. Anais. v.2. Recife. [s/p]. 2008. 